\title{
Sulfidation of Cadmium at the Nanoscale
}

\author{
Andreu Cabot, ${ }^{1}$ Rachel K. Smith, ${ }^{1}$ Yadong Yin, ${ }^{2}$ Haimei Zheng, ${ }^{1}$ Björn M. \\ Reinhard, ${ }^{3}$ Haitao Liu, ${ }^{4}$ and A. Paul Alivisatos ${ }^{1,4 * *}$ \\ ${ }^{1}$ Materials Science Division, Lawrence Berkeley National Laboratory, Berkeley, CA 94720. \\ ${ }^{2}$ Department of Chemistry, University of California at Riverside, Riverside, CA 92521. \\ ${ }^{3}$ Department of Chemistry, Boston University, Boston, MA 02215. \\ ${ }^{4}$ Department of Chemistry, University of California at Berkeley, Berkeley, CA 94720. \\ *Address correspondence to: alivis@ berkeley.edu.
}

\begin{abstract}
We investigate the evolution of structures that result when spherical $\mathrm{Cd}$ nanoparticles of a few hundred nanometers in diameter react with dissolved molecular sulfur species in solution to form hollow CdS. Over a wide range of temperatures and concentrations, we find that rapid $\mathrm{Cd}$ diffusion through the growing $\mathrm{CdS}$ shell localizes the reaction front at the outermost $\mathrm{CdS} / \mathrm{S}$ interface, leading to hollow particles when all the $\mathrm{Cd}$ is consumed. When we examine partially reacted particles, we find that this system differs significantly from others in which the nanoscale Kirkendall effect has been used to create hollow particles. In previously reported systems, partial reaction creates a hollow particle with a spherically symmetric metal core connected to the outer shell by filaments. In contrast, here we obtain a lower symmetry structure, in which the unreacted metal core and the coalesced vacancies separate into two distinct spherical caps, minimizing the metal/void interface. This pattern of void coalescence is likely to occur in situations where the metal/vacancy self-diffusivities in the core are greater than the diffusivity of the cations through the shell.
\end{abstract}

Keywords: hollow nanoparticle, Kirkendall effect, self-diffusion, diffusion mechanism, CdS, sulfidation 
Since the first report in $2004,{ }^{1}$ the formation of hollow nanoparticles via the nanoscale Kirkendall effect has been observed in dozens of materials. ${ }^{2-5}$ In the simplest scenario, an elemental metallic nanocrystal reacts to form a compound nanoparticle; hollow, polycrystalline nanoparticles result when the diffusion of the cationic species outwards is faster than the inward diffusion of the anionic species. Some examples of hollow particles formed in this way include oxides, sulfides, selenides, and phosphides of cobalt, iron, molybdenum, and nickel. Fan, Gösele, and Zacharias have written a recent review outlining developments in this field, ${ }^{2}$ as well as describing criteria that can be used to evaluate the possibility that a given system will form hollow particles by this mechanism.

In materials with one or more macroscopic dimensions, like nanowires or films, the Kirkendall effect leads to the formation of multiple voids at the metal-composite interface. ${ }^{6,7}$ In nanoparticles, a single void is typically observed in the center of the particle when the reaction is complete, and the particles maintain an overall approximate spherical symmetry through the course of the reaction. ${ }^{3,} 8$ In such spherical nanostructures, the unreacted metal core remains in the center and is connected to the hollow shell by thin filaments. ${ }^{1,9}$ In a very recent report, Nakamura et al. already showed that the incomplete oxidation of $\mathrm{Ni}$ nanoparticles leads to the formation of partially hollow structures with a single void. ${ }^{10}$ On fully oxidized $\mathrm{NiO}$ particles, holes were located at off-center positions and non-homogeneous shell thicknesses were obtained. Here we explore the sulfidation of spherical cadmium crystals to form hollow CdS via the nanoscale Kirkendall effect, Figure 1. Cadmium sulfide, having both direct band gaps in the optical region and piezoelectric properties, is particularly suited for optical, optoelectronic, and electromechanical applications. In the cadmium sulfidation reaction, the metal self-diffusion time in the core is much lower than the self-diffusion time of the metal ions through the shell. We show that the structures obtained from the partial sulfidation of $\mathrm{Cd}$ particles contain a single off-center void region and a single off-center region of unreacted $\mathrm{Cd}$, both with a spherical cap geometry. Markedly, in spite of the asymmetrical metal distribution in this new type of structure, a homogeneous growth of the CdS shell is observed throughout the entire particle as the sulfidation proceeds to completion. We measured the growth rates of $\mathrm{CdS}$ as a function of the reaction 
temperature and the sulfur concentration, and have estimated the Cd self-diffusivity in the $\mathrm{CdS}$ shells. This estimate allows for a self-consistent explanation of the mechanism of formation of the $\mathrm{Cd} / \mathrm{CdS}$ heterostructures; the calculated diffusion coefficient is used to discriminate between the mechanisms of Cd transport through the CdS shell.

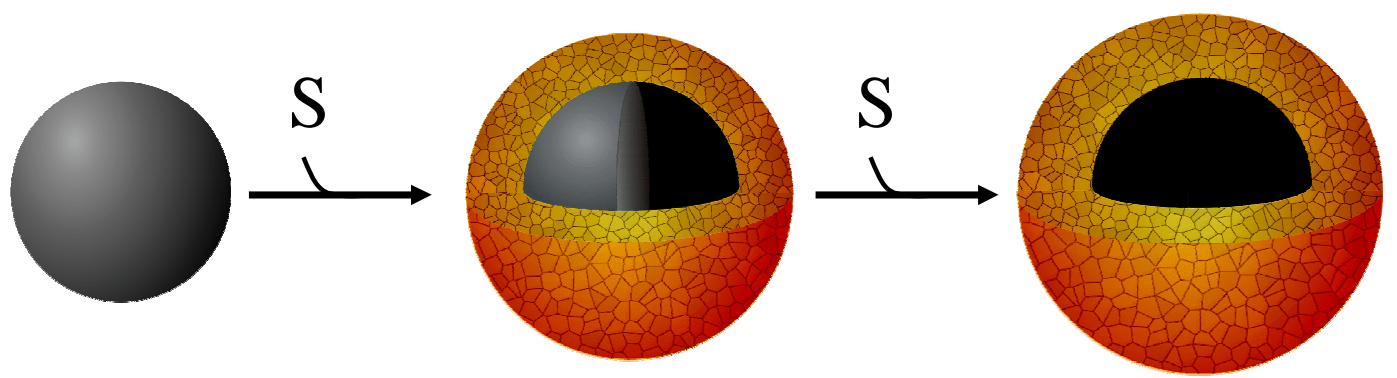

Figure 1. Schematic of the sulfidation process of $\mathrm{Cd}$ nanoparticles. Solid cadmium particles (left) are exposed to elemental sulfur leading to partially sulfidized structures, with a $\mathrm{Cd}$ core remaining inside a polycrystalline $\mathrm{Cd}$ sulfide shell (center). Longer reaction times lead to fully hollow CdS structures (right).

\section{RESULTS AND DISCUSSION}

Solid cadmium particles were synthesized by the decomposition of dimethylcadmium in trioctylphosphine oxide (TOPO) at $330{ }^{\circ} \mathrm{C}$ under air-free conditions. In the absence of oxidizing elements, dimethylcadmium decomposes to form colloidal $\mathrm{Cd}^{0}$. At temperatures below the melting point of cadmium $\left(\mathrm{T}_{\mathrm{m}}=321{ }^{\circ} \mathrm{C}\right)$, cadmium particles appear highly faceted, having hexagonal geometries. Above its melting point, cadmium forms spherical droplets in the liquid phase, and these solidify into spherical Cd particles as the temperature is decreased. The size of the cadmium particles can be tuned in the diameter range between $100 \mathrm{~nm}$ and $1 \mu \mathrm{m}$ by the concentration of the injected solution and the stirring rate. ${ }^{11}$ The resulting $\mathrm{Cd}$ nanoparticles are monocrystalline, as deduced from both electron diffraction and dark field transmission electron microscopy (TEM) (Supporting Information, Figure 1).

Sulfidation of colloidal Cd crystals was performed by the injection of elemental sulfur dissolved in 1,2-dichlorobenzene into a solution of colloidal Cd. Sulfidation was carried out in the temperature range of $200-300{ }^{\circ} \mathrm{C}$, and with sulfur to cadmium ratios $([\mathrm{S}] /[\mathrm{Cd}])$ in the range of 1-12. The actual concentration of sulfur in solution will be 
lower than the nominal concentration injected, as a perceptible percentage of sulfur may react with the solvent impurities. Moreover, a slight reduction of the sulfur concentration with time is to be expected from the combination of the high vapor pressure of sulfur at the reaction temperature $(\sim 1 \mathrm{kPa})$ and the use of continuous flow of argon in the experimental set-up.

Transmission electron microscopy was used to follow the progressive sulfidation of the spherical Cd particles, Figure 2. We observe that, upon sulfur introduction, a uniform and polycrystalline $\mathrm{CdS}$ shell forms at the outer surface of the $\mathrm{Cd}$ particle, and as the sulfidation proceeds, the shell grows outward from the CdS/solution interface, as observed from the evolution of the thickness and the outer diameters of the shell, Figure 3. This external growth requires the diffusion of $\mathrm{Cd}$ through the $\mathrm{CdS}$ shell and therefore, an inward diffusion of the equivalent number of vacancies follows to counterbalance the outward diffusion of matter. In the initial stages of $\mathrm{Cd}$ sulfidation, vacancies coalesce into voids extending all over the $\mathrm{Cd} / \mathrm{CdS}$ interface, Figure 2B. However, in a slightly advanced stage of sulfidation, vacancies are found to coalesce into a single void with spherical cap shape adhering to one side of the interior hollow CdS shell, Figures 2C-E. In spite of this asymmetric $\mathrm{Cd}$ distribution, the spherical $\mathrm{CdS}$ shell still grows isotropically. Tilting of the sample holder inside the TEM allows us to confirm that the $\mathrm{Cd}$ in the partially converted particles assembles in a spherical cap shape, Figure 4. As the sulfidation proceeds, the $\mathrm{Cd}$ core is consumed through its free interface in a similar way as the liquid level in a container is reduced while preserving a flat surface. However, the intuitive image of a molten $\mathrm{Cd}$ core inside the shell at the reaction temperature, which then solidifies upon cooling, has been definitively ruled out, using differential scanning calorimetry (DSC) analysis of the intermediate heterostructures. In Figure 5, DSC data show that the melting temperature of the spherical Cd caps inside the $\mathrm{CdS}$ shell is approximately equal to that of bulk $\mathrm{Cd}\left(321^{\circ} \mathrm{C}\right)$, well above the sulfidation temperatures used $\left(200-300{ }^{\circ} \mathrm{C}\right)$. 

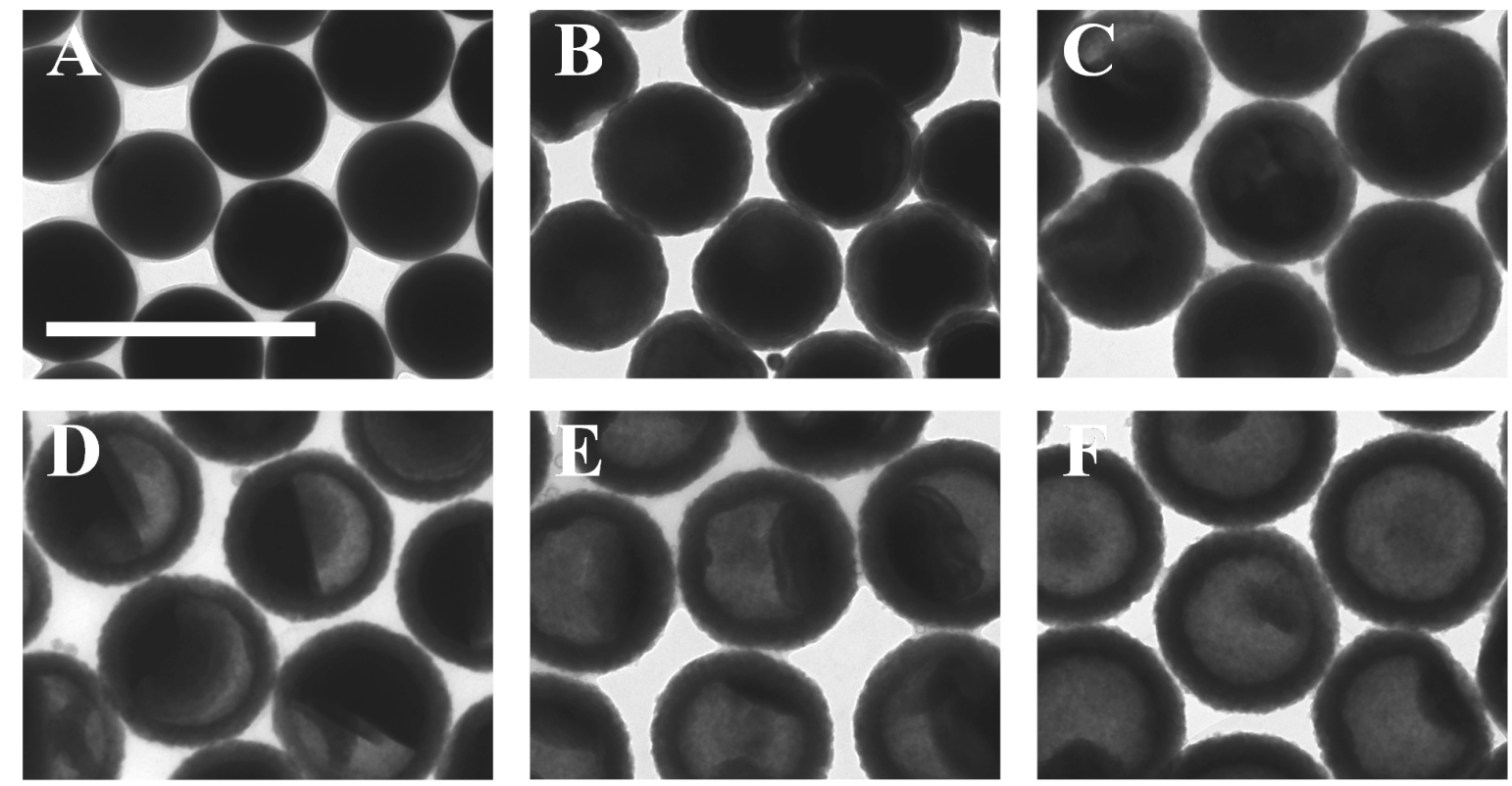

Figure 2. TEM micrographs from 6 aliquots at different stages of the CdS shell growth; from the initial $\mathrm{Cd}$ particles (A) to the final CdS hollow particles (F). The scale bar corresponds to $500 \mathrm{~nm}$.

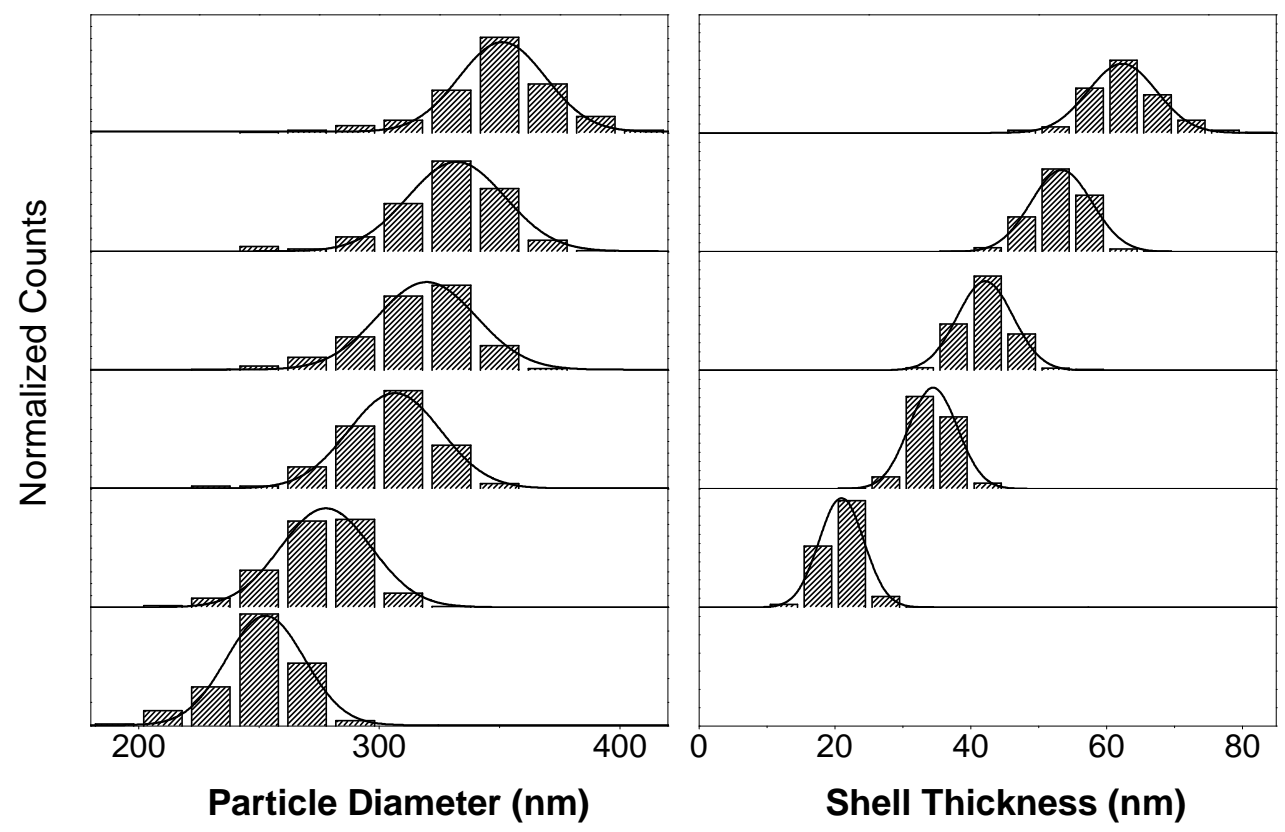

Figure 3. Particle diameter and shell thickness distributions from 6 aliquots at different stages of the CdS shell growth: from the initial Cd particles (bottom histograms) to the final CdS hollow particles (top histograms). 

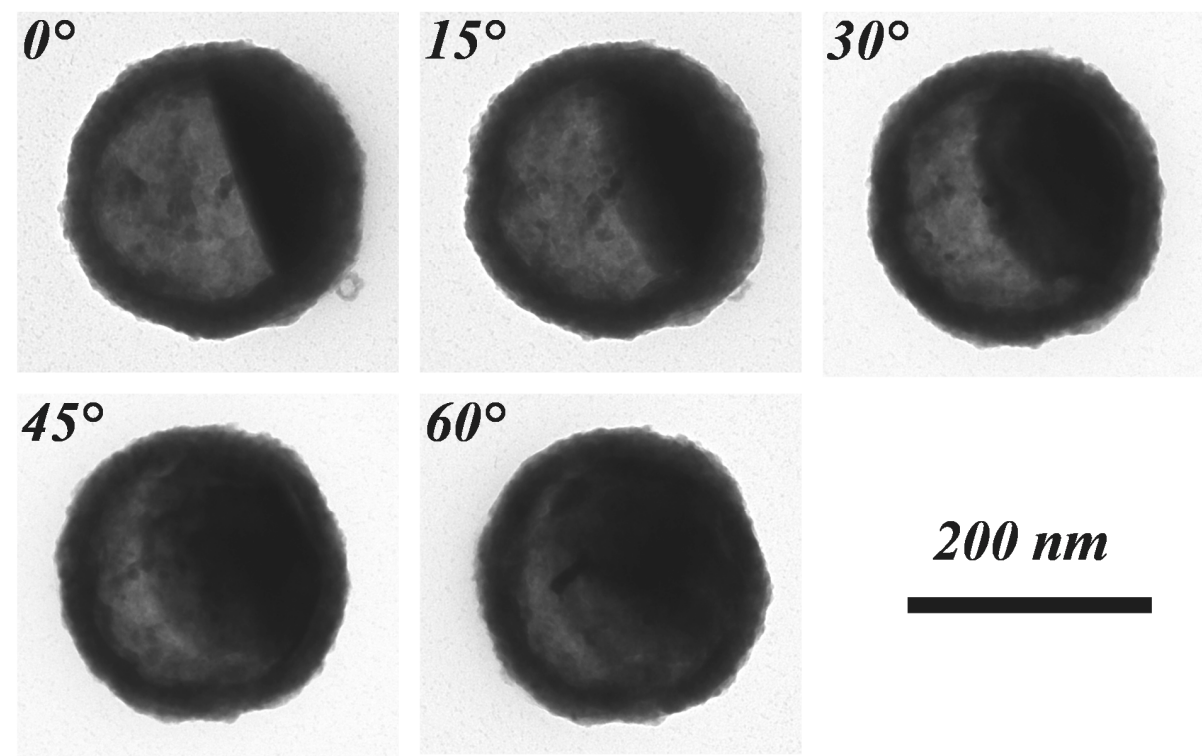

\section{$200 \mathrm{~nm}$}

Figure 4. TEM micrographs at different tilting angles of the same partially reacted $\mathrm{Cd} / \mathrm{CdS}$ particle.

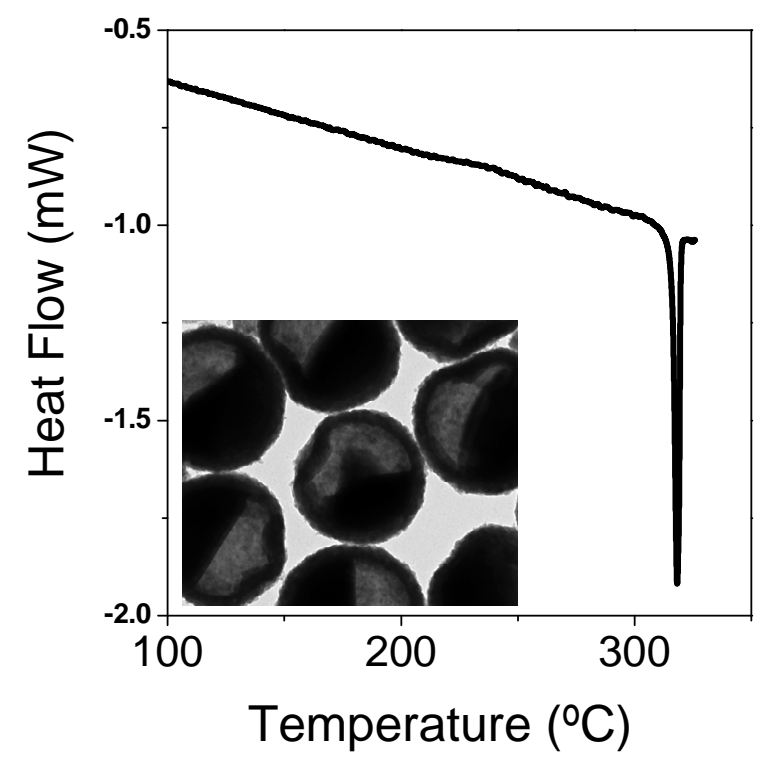

Figure 5. Differential scanning calorimetry (DSC) analysis of the intermediate $\mathrm{Cd} / \mathrm{CdS}$ particles. The endothermic peak at $\sim 320^{\circ} \mathrm{C}$ corresponds to the melting of the $\mathrm{Cd}$ cores inside of the CdS shells. Inset: TEM micrograph of the partially hollow $\mathrm{Cd} / \mathrm{CdS}$ nanoparticles analyzed by DSC. 
It is interesting to consider the complex balance of thermodynamic and kinetic factors at play in the formation of the spherical caps during the partial sulfidation. Three interface energies play a critical role in this system: core/shell, core/void, and void/shell. Here, the coalescence of the $\mathrm{Cd}$ into a single spherical cap minimizes the interface between the metal and the surrounding void/medium, ${ }^{12}$ Figure 5 . This indicates that the metal/void interface is the highest energy interface in the system. Both Cd cations and vacancies must diffuse quickly through the shell to account for the homogeneous feeding of the growing $\mathrm{CdS}$ shell. The geometry of the metal core in partially oxidized particles can be thermodynamically directed only for long enough reaction times, as compared to the diffusion time of the core element. If we consider the reaction time to be limited by the diffusivity of the metal through the shell, this condition can be expressed as a ratio of self-diffusivities. Consider Arrhenius expressions for both self-diffusivities, with $D_{0 S}$, $D_{0 C}, E_{C}$ and $E_{S}$ being the self-diffusivity constants and activation energies of the metal or metal ions through in the core and the shell. The condition for the formation of metal spherical caps instead of bridge-supported cores can be expressed as the following rate between the metal diffusion time in the core $\left(t_{C}\right)$ and its diffusion time through the shell $\left(t_{S}\right)$ :

$$
\frac{t_{C}}{t_{S}}=A \cdot \frac{D_{0 S}}{D_{0 C}} \cdot \exp \left(\frac{E_{C}-E_{S}}{k T}\right)<1
$$

where:

$$
A=\frac{1}{\left((1+\theta)^{1 / 3}-1\right)}
$$

accounts for the different diffusion length of the metal in the core and the ions in the shell, $\theta$ being the molar volume ratio between the initial and final structure, also known as the Pilling-Bedworth ratio.

Extrapolation of self-diffusivity values previously reported in the literature suggests that the sulfidation of cadmium should indeed satisfy the conditions of equation (1). The Cd self-diffusivity is estimated to be much faster in the core than through the CdS shell (Table 1). This is consistent with our experimental results, where the metal 
keeps a thermodynamically favorable shape while supporting an effective and uniform sulfidation reaction. The same argument applies for the oxidation of $\mathrm{Zn}$ particles, for example. On the other hand, in the sulfidation and oxidation of Co, and in the oxidation of $\mathrm{Fe}$, the faster self-diffusion of ions through the shell than in the core, leads to the formation of kinetically controlled $\mathrm{Co} / \mathrm{Co}_{\mathrm{x}} \mathrm{S}_{\mathrm{y}}$ and $\mathrm{Fe} / \mathrm{Fe}_{\mathrm{x}} \mathrm{O}_{\mathrm{y}}$ nanostructures, with spherical cores supported by bridges, and with very high metal/void interface areas. ${ }^{1,3}$

Table 1. Metal Diffusion Coefficients $\left[D=D_{0} \exp (-E / k T)\right] .^{13}$

\begin{tabular}{cccccc}
\hline Element & $\boldsymbol{T}_{\boldsymbol{M}}\left({ }^{\circ} \boldsymbol{C}\right)^{\boldsymbol{a}}$ & $\boldsymbol{D}_{\boldsymbol{0}}\left(\mathrm{cm}^{2} \boldsymbol{s}^{-1}\right)$ & $\boldsymbol{E}(\boldsymbol{e V})$ & $\boldsymbol{T}\left({ }^{\circ} \boldsymbol{C}\right)^{b}$ & $\boldsymbol{D}_{200}\left(\mathbf{n m}^{2} \boldsymbol{s}^{-1}\right)^{\boldsymbol{c}}$ \\
\hline In & 157 & $3-4$ & 0.8 & $39-144$ & $\sim 10^{5}\left(T=150^{\circ} \mathrm{C}\right)$ \\
$\mathrm{Sn}$ & 232 & $1-20$ & $1.0-1.1$ & $160-228$ & $\sim 10^{3}$ \\
$\mathrm{Cd}$ & 321 & $0.05-0.7$ & $0.8-0.9$ & $77-315$ & $\sim 10^{4}$ \\
$\mathrm{Zn}$ & 420 & $0.1-0.6$ & $0.9-1.1$ & $240-418$ & $\sim 10^{2}-10^{3}$ \\
$\mathrm{Al}$ & 660 & $0.1-2$ & $1.3-1.5$ & $85-643$ & $\sim 10^{-2}-10^{-1}$ \\
$\mathrm{Cu}$ & 1085 & $0.1-1$ & $2.0-2.2$ & $301-1082$ & $\sim 10^{-10}-10^{-8}$ \\
$\mathrm{Ni}$ & 1455 & $1-3$ & $2.9-3.1$ & $475-1404$ & $\sim 10^{-8}-10^{-7}$ \\
$\mathrm{Co}$ & 1495 & $0.2-2$ & $2.7-3.2$ & $623-1472$ & $\sim 10^{-19}-10^{-16}$ \\
$\mathrm{Fe}$ & 1538 & $0.2-10$ & $2.5-3.2$ & $697-1508$ & $\sim 10^{-20}-10^{-12}$ \\
$\mathrm{Ti}$ & 1668 & $10^{-7}-10^{-3}$ & $1.3-1.8$ & $690-1583$ & $\sim 10^{-9}-10^{-4}$ \\
\hline
\end{tabular}

${ }^{a}$ Melting temperature;

${ }^{b}$ Temperature range of the measurement;

${ }^{c}$ Extrapolation of the diffusion coefficient to $200{ }^{\circ} \mathrm{C}$.

The self-diffusivity values reported in the literature are measured at temperatures well above those used here, and using macroscopic crystals. Diffusivities strongly depend not only on the temperature but also on the microstructure, composition, and potentially also the overall dimension of the sample. Thus, the self-diffusion values extrapolated from literature values may differ significantly from those in the polycrystalline shells and at the temperatures considered here. Furthermore, the ion self-diffusivity may not be the limiting parameter on the growth/reaction rate, but the reaction time may be much larger 
due for example to a limited supply of the oxidizing element in solution. Here we show that a more comprehensive study of the hollow nanoparticle evolution itself can be used to provide a direct measure of some of the self-diffusivities at work in this experiment.

The present system offers the opportunity to directly measure the kinetics of the sulfidation process at the nanoscale. X-ray diffraction (XRD) was used to quantitatively follow the sulfidation process by removing aliquots at successive times after the sulfur injection, Figure 6. The CdS shell growth rate can be calculated by monitoring the appearance of $\mathrm{CdS}$ as a function of time with a concomitant decrease in the $\mathrm{Cd}$. Figure 7A shows the fraction of $\mathrm{CdS}$ obtained from the reaction of $350 \mathrm{~nm} \mathrm{Cd}$ particles with different nominal sulfur concentrations. It is striking that the reaction rate is strongly affected by small changes in the amount of added sulfur only at low sulfur concentrations. At the crossover of reaction regimes the sulfur to cadmium ratio is $[S] /[\mathrm{Cd}]=6$. At lower sulfur concentrations, we believe the sulfur reaction probability or its diffusion to control the reaction rate. In order to discern between these two mechanisms, we can obtain the order of magnitude of the sulfur reactivity. We consider the most abundant molecular form of sulfur in solution to be $\mathrm{S}_{8}{ }^{14}$ The approximated diffusion constant of these molecules, as given by the Stokes-Einstein relation with a solvent mobility of $0.1 \mathrm{~Pa} \cdot \mathrm{s}$, is on the order of $10^{7} \mathrm{~nm}^{2} \mathrm{~s}^{-1}$. At a sulfur to cadmium ratio of $[\mathrm{S}] /[\mathrm{Cd}]=4$, the complete sulfidation of $350 \mathrm{~nm} \mathrm{Cd}$ particles takes place in around 40 $\min$ at $240{ }^{\circ} \mathrm{C}$. This sulfur concentration in solution, $\left[\mathrm{S}_{8}\right] \sim 0.02 \mathrm{~nm}^{-3}$, translates into a collision rate of around $6 \times 10^{8} \mathrm{~nm}^{-2} \mathrm{~s}^{-1}$. From the sulfidation time, we deduce that the flux of $\mathrm{Cd}$ through the shell is around 2 atoms $\mathrm{nm}^{-2} \mathrm{~s}^{-1}$. Thus the reaction probability of the $\mathrm{S}_{8}$ molecules probing the surface of the $\mathrm{CdS}$ with the diffusing $\mathrm{Cd}$ atoms is on the order of $10^{-7}$. From this estimation of the order of magnitude of the reaction probability, we conclude that the concentration of sulfur around the particles does not perceptibly change because of its reaction with the $\mathrm{Cd}$, which rules out the $\mathrm{S}$ diffusivity as the limiting rate factor. It is therefore the low reaction probability that controls the rate of sulfidation of the Cd particles at sulfur concentrations lower than $[\mathrm{S}] /[\mathrm{Cd}]=6$. 


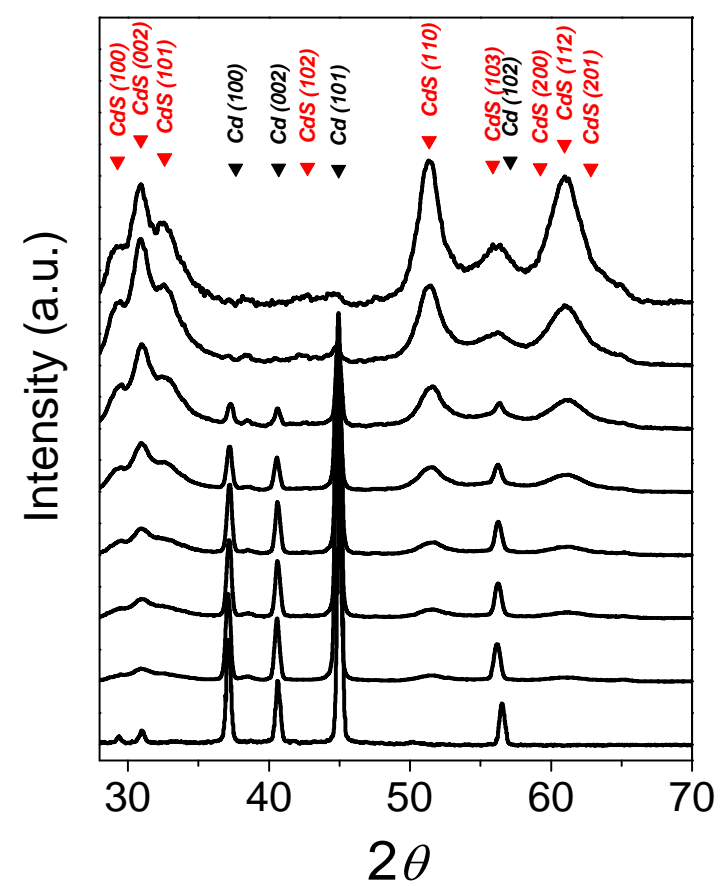

Figure 6. Evolution of the X-ray diffraction patterns of the hollow CdS particles, from the initial Cd particles (bottom pattern) to the final CdS hollow particles (top pattern).
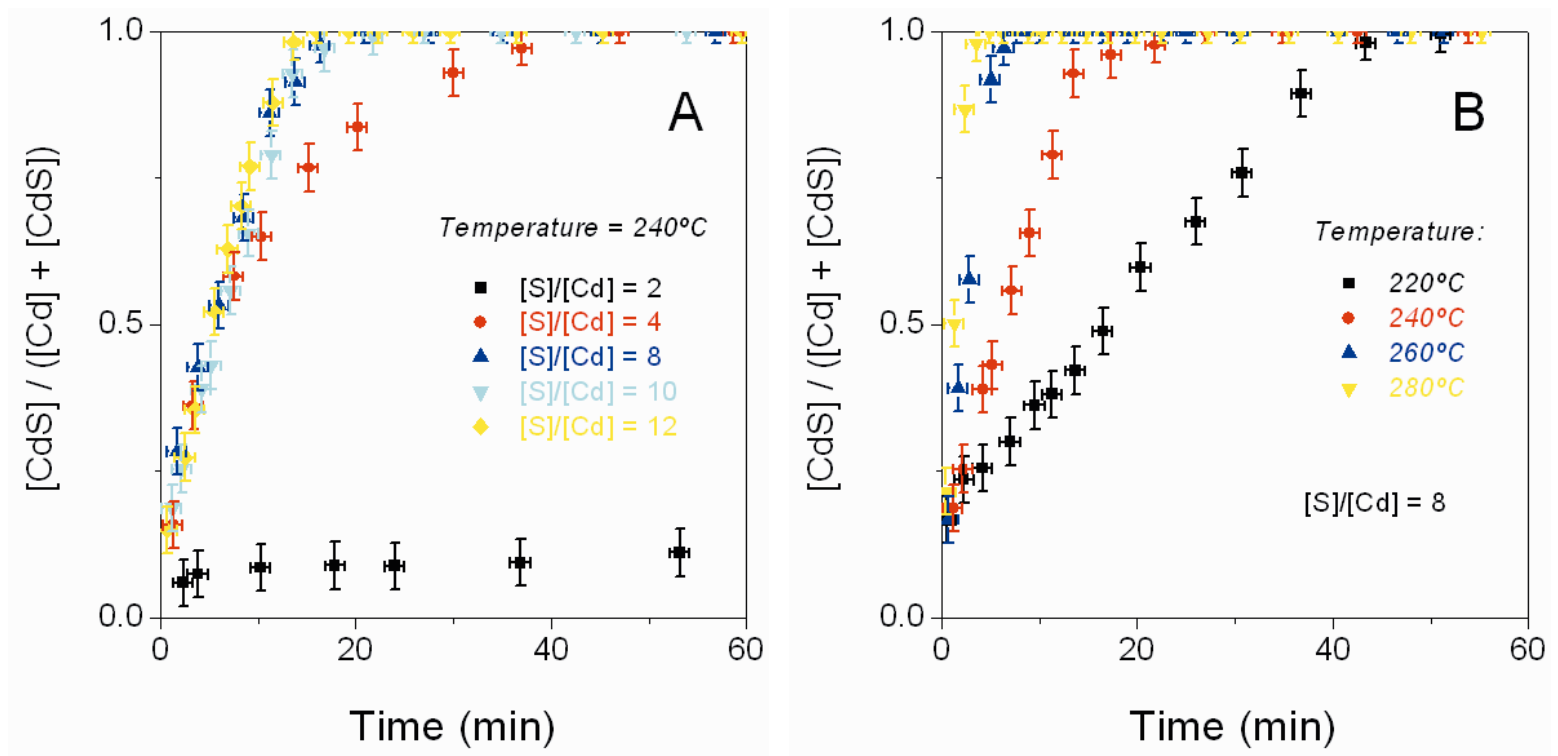

Figure 7. Time evolution of the fraction of CdS formed from the reaction of $350 \mathrm{~nm}$ cadmium particles. (A) The reaction temperature is set to $240{ }^{\circ} \mathrm{C}$, while the nominal sulfur concentration is changed as specified in the graph. (B) The nominal sulfur 
concentration is set to $[\mathrm{S}] /[\mathrm{Cd}]=8$, while the reaction temperature is changed as specified in the graph.

At higher sulfur concentrations, the shell growth rate is independent of the amount of sulfur in solution. In this reaction regime, the collision frequency is large enough to lead to the prompt reaction of every $\mathrm{Cd}$ reaching the shell outer surface, even though the reaction probability is low. This is confirmed by using TEM to monitor in situ the disappearance of the $\mathrm{Cd}$ core when heated in high vacuum, Figure 8. TEM studies confirm that the Cd core disappears on the same time scale as the growth of the shell. As noted above, the Cd self-diffusion inside the void is very fast when compared with the shell growth rate (Table 1). Thus, we consider the Cd diffusivity through the shell, either in a neutral or ionized form, as the limiting rate in this reaction regime.
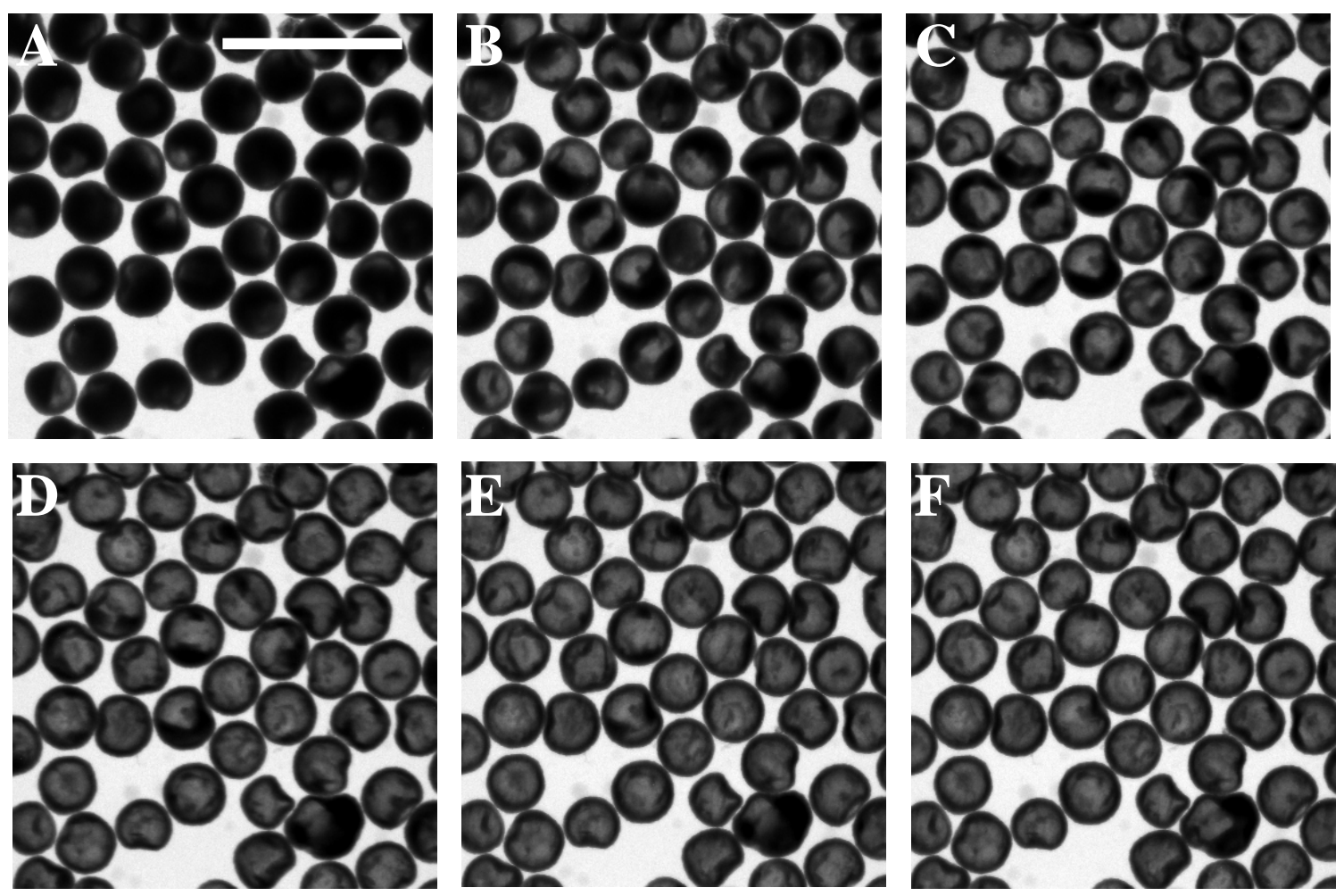

Figure 8. TEM micrographs of different stages of the vaporization of the cadmium cores inside thin $\mathrm{CdS}$ shells at $285^{\circ} \mathrm{C}$. Scale bar corresponds to $1 \mu \mathrm{m}$.

The oxidation kinetics of metal films controlled by the diffusion of one of the reactants usually results in parabolic rates. By considering the diffusivity of one of the 
ionized reactants to be driven by local electric fields, Cabrera and Mott have estimated the following parabolic dependence of a metal oxide film growth rate: ${ }^{15}$

$$
l^{2}=4 D_{i} \Omega[n(0)-n(l)] t
$$

where $l$ is the thickness of the grown film, $D_{i}$ is the diffusion coefficient for metal ions, $\Omega$ is the volume of oxide grown per metal ion, and $n(0)-n(l)$ is the difference in the concentration of dissolved diffusing ions between the inner and the outer surface of the film. A similar dependence can be derived when considering the diffusion of neutral species as being driven by concentration gradients. From the general problem of diffusion with discontinuous boundaries, solved by Hermans for the specific case of diffusion with precipitation, ${ }^{16}$ the following dependence is obtained:

$$
l^{2}=4 B^{2} D t
$$

where $D$ is the diffusion coefficient of the diffusing substance in the medium, and $B$ is given by:

$$
B e^{B^{2}} \operatorname{erf}(B)=\frac{a}{\sqrt{\pi} b}
$$

with $a$ being the concentration of diffusing particles at $x=0$, and $b$ as the concentration of binding sites.

The time evolution of the $\mathrm{Cd}$ to $\mathrm{CdS}$ conversion obtained from the reaction of 350 $\mathrm{nm} \mathrm{Cd}$ particles with a nominal sulfur concentration $[\mathrm{S}] /[\mathrm{Cd}]=8$ is shown in Figure 7B. In Figure 9, the shell thickness is calculated from the measured $\mathrm{CdS}$ fraction and is plotted as a function of the square root of the reaction time at different reaction temperatures. At low reaction times a linear dependence, which flattens at high $\mathrm{CdS}: \mathrm{Cd}$ ratios, is obtained. 


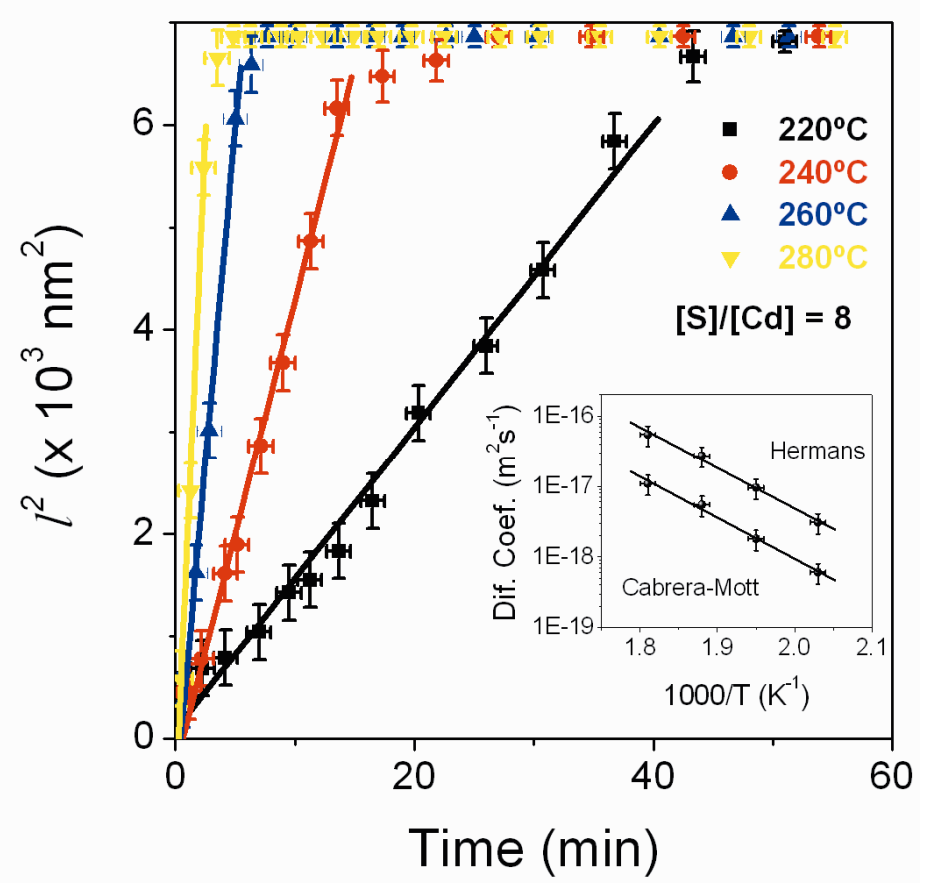

Figure 9. Time dependence of the square of the shell thickness for different reaction temperatures: $220{ }^{\circ} \mathrm{C}$ (black), $240{ }^{\circ} \mathrm{C}$ (red), $260{ }^{\circ} \mathrm{C}$ (green) and $280{ }^{\circ} \mathrm{C}$ (blue). The nominal concentration of sulfur is set to a ratio $[\mathrm{S}] /[\mathrm{Cd}]=8$. The lines correspond to linear fittings of the data points. The inset shows the Arrhenius plot of the obtained diffusion coefficients for the two models described in the text.

The linear data regimes in Figure 9 were fitted to expressions (1) and (2). The reasonably good fit of the data obtained from the growth of spherical shells to the expressions describing growth rates in planar geometry is not surprising when considering the moderate increase in the particle diameter with the oxidation process. However, a better estimation of the diffusion coefficients by this method should introduce corrections for this spherical geometry.

To determine the diffusion coefficient from these fits, the concentration of dissolved cadmium in the inner surface was approximated to the concentration of $\mathrm{Cd}$ in the CdS lattice. Both Cabrera-Mott and Hermans models, considering charged or neutral diffusing species respectively, lead to very similar $\mathrm{Cd}$ self-diffusivities with identical 
temperature dependences (inset, Figure 9). From the diffusion coefficients obtained for the different reaction temperatures, the following estimation of the Arrhenius dependence of the Cd diffusion coefficient $\left(\mathrm{m}^{2} \mathrm{~s}^{-1}\right)$ was obtained:

$$
D=D_{0} \exp (-E / k T)=1 \times 10^{-6} \exp (1.1 \mathrm{eV} / \mathrm{kT})
$$

The values reported in the literature for the diffusivity of $\mathrm{Cd}$ and $\mathrm{S}$ in $\mathrm{CdS}$ crystal lattices are compared to those obtained in the present work in Table 2. The pre-exponential factor, $D_{0}$, obtained here is inside the large range of values previously measured for a variety of systems. The activation energy calculated in the present work is just half of that reported in previous studies. Such low activation energy is consistent with the polycrystalline nature of the CdS shells. It is well known that diffusion coefficients in nanocrystalline materials can be orders of magnitude larger than in their bulk counterparts. ${ }^{17}$ Grain boundaries provide easy paths for ion movement, and thus activation energies for grain boundary diffusion are often much lower than those for intrinsic bulk lattice diffusion. ${ }^{18,} 19$ Therefore, our studies confirm grain boundary diffusion of ionized or neutral cadmium as the dominant mechanism for cadmium transport through the shell. This diffusion mechanism is associated with a strong dependence of the growth rate on the shell nanostructure. Furthermore, the Cd diffusivity through the shell obtained by our estimation is approximately four orders of magnitude slower than the cadmium self-diffusivity. We believe this large difference to be the reason behind the thermodynamic accommodation of the core inside the shell.

Table 2. Cd and S Self-Diffusion Coefficients in CdS $\left[D=D_{0} \exp (-E / k T)\right] .^{20}$

\begin{tabular}{|c|c|c|c|c|}
\hline Reference & $\begin{array}{c}\text { Diffusing } \\
\text { Element }\end{array}$ & $T(K)$ & $D_{0}\left(m^{2} s^{-1}\right)$ & $E(e V)$ \\
\hline Present work & $\mathrm{Cd}$ & $493-553$ & $1 \times 10^{-6}$ & 1.1 \\
\hline H. H. Woodbury ${ }^{21}$ & $\mathrm{Cd}$ & $793-1373$ & $3.4 \times 10^{-4}$ & 2.0 \\
\hline D. Shaw ${ }^{22}$ & $\mathrm{Cd}$ & $773-973$ & $6.7 \times 10^{-2}$ & 2.67 \\
\hline D. Shaw ${ }^{22}$ & $\mathrm{Cd}$ & $973-1123$ & $5.8 \times 10^{-6}$ & 1.85 \\
\hline E. D. Jones ${ }^{23}$ & $\mathrm{Cd}$ & $875-1528$ & $1.2 \times 10^{-4}$ & 2.3 \\
\hline
\end{tabular}




\begin{tabular}{|c|c|c|c|c|}
\hline V. Kumar and F. A. Kroger ${ }^{24}$ & $\mathrm{Cd}$ & $973-1273$ & $3.3 \times 10^{-6}$ & 2.0 \\
\hline E. D. Jones et al. ${ }^{25}$ & $\mathrm{Cd}$ & $1053-1550$ & $4.2 \times 10^{-6}$ & 1.90 \\
\hline E. D. Jones et al. ${ }^{25}$ & $\mathrm{Cd}$ & $870-1053$ & 5.2 & 3.18 \\
\hline L. A. Sysoev et al. ${ }^{26}$ & $\mathrm{Cd}$ & $1023-1323$ & & 2.4 \\
\hline V. Kumar and F. A. Kroger ${ }^{24}$ & $\mathrm{~S}$ & $1073-1173$ & $1.6 \times 10^{-6}$ & 2.08 \\
\hline
\end{tabular}

\section{Conclusions}

We have investigated the sulfidation reaction of $350 \mathrm{~nm} \mathrm{Cd}$ particles. Our studies reveal that $\mathrm{Cd}$ diffuses more rapidly than $\mathrm{S}$ through the polycrystalline shells that result from the reaction of $\mathrm{Cd}$ with $\mathrm{S}$. This high $\mathrm{Cd}$ diffusivity leads to the accumulation of vacancies at the metal/sulfide interface, and eventually to the de-cohesion of the growing $\mathrm{CdS}$ shell. Through control of the reaction time, reaction temperature, and $\mathrm{Cd} / \mathrm{S}$ ratio, the particle morphology could be shifted from metal-void-shell structures containing the unreacted metal localized in a single core attached to the $\mathrm{CdS}$ shell, to completely hollow $\mathrm{CdS}$ structures. In contrast to previously described systems, here in partially sulfided $\mathrm{Cd}$ particles, a single Cd core is observed and its interface with the void is minimized with a spherical cap geometry. This particular arrangement of the core metal is explained by the different time scales of $\mathrm{Cd}$ diffusion inside the particle and across the shell. Such a system offers a unique opportunity to estimate diffusion coefficients at relatively low temperatures. At high sulfur concentrations, the growth rate is limited by the $\mathrm{Cd}$ diffusion rate through the shell, which was estimated to be $D=1 \times 10^{-6} \exp (1.1 \mathrm{eV} / \mathrm{kT})$. The activation energy obtained from this approach is lower than that measured at higher temperatures for lattice diffusion. This deviation is consistent with the polycrystalline nature of the formed CdS shells. At the same time, the estimated Cd self-diffusion in the CdS shells is much lower than the Cd metal self-diffusion. This is consistent with the considered mechanism controlling the unreacted metal geometry and the simultaneous homogeneous growth of the shell. 


\section{METHODS}

Synthesis. Solid cadmium particles were obtained by decomposition of dimethylcadmium (97\%, Strem) in trioctylphosphine oxide (TOPO, 99\%, SigmaAldrich). Dimethylcadmium was distilled prior to use, and stored below its melting point in an argon-filled glovebox. In a typical synthesis, $8 \mathrm{~g}$ of TOPO were heated inside a three-neck flask to $60^{\circ} \mathrm{C}$ under vacuum for $30 \mathrm{~min}$. While keeping the solution under argon $(99.999 \%)$, the temperature was raised to $330^{\circ} \mathrm{C}$. A precursor solution of $0.1 \mathrm{~g}$ of dimethylcadmium in $1 \mathrm{ml}$ of trioctylphosphine (TOP, 97\%, Strem) was prepared separately under Ar. This mixture was rapidly injected through a septum into the hot solution with vigorous stirring. The decomposition of the dimethylcadmium is instantaneous, and leads to the formation of colloidal $\mathrm{Cd}$ particles. When formed above the cadmium melting temperature $\left(\mathrm{T}_{\mathrm{m}}=321{ }^{\circ} \mathrm{C}\right)$, these $\mathrm{Cd}$ particles have a spherical symmetry. Following the injection, the temperature dropped to around $320{ }^{\circ} \mathrm{C}$, and then slowly recovered to $330{ }^{\circ} \mathrm{C}$. The solution was kept at a temperature between $320{ }^{\circ} \mathrm{C}$ and $330^{\circ} \mathrm{C}$ for around 30 s, and was then rapidly decreased to quench the spherical geometry of the liquid drops.

To sulfidize the colloidal Cd crystals, a 30\% sulfur solution was prepared inside the glovebox by dissolving $0.4 \mathrm{~g}$ of elemental sulfur $(99.998 \%$, Aldrich) in $10 \mathrm{~mL}$ of anhydrous 1,2-dichlorobenzene (99\%, Aldrich). Dissolution was carried out using a heated ultrasound sonicator, while keeping the sample closed in air-free conditions inside a vial accessible through a septum. The sulfur solution has a dark-brown color. Between $0.5 \mathrm{~mL}$ and $6.7 \mathrm{~mL}$ of this solution were injected through a septum into the hot solution containing the $\mathrm{Cd}$ crystals. The reaction temperature was set between $200^{\circ} \mathrm{C}$ and $300^{\circ} \mathrm{C}$. After injection, the temperature drops a certain amount, depending on the injected volume, but is quickly raised as part of the injected dichlorobenzene is removed by evaporation. The sulfidation requires anywhere from a few minutes to hours, and can be qualitatively monitored from the solution's change in color from grey to orange.

Characterization. The crystal structure of the samples was characterized using powder X-ray diffraction. XRD analyses were obtained on a Bruker AXS diffractometer using Co

Ka radiation (1.79026 ̊) and a general area detector. The instrument resolution was 
$0.05^{\circ}$ in $2 \theta$, and the acquisition time for each sample was around 1 hour. XRD samples were prepared in air by depositing the precipitated particles on a quartz substrate. XRD was also used to quantitatively follow the sulfidation process. $0.3 \mathrm{~mL}$ aliquots were removed through the septum at successive times after the sulfur injection. These aliquots were immediately dissolved in chloroform at room temperature and cleaned by centrifugation to remove excess sulfur in solution. The dried samples were immediately characterized by means of XRD and TEM. For the purpose of a quantitative analysis of the cadmium to cadmium sulfide conversion, we integrated the XRD diffraction patterns obtained from the analysis of the successive aliquots. We compared the area of the $\mathrm{CdS}$ (100), (002) and (101) diffraction peaks with that of the (101) Cd peak. A sample containing a $50 \%$ of as-synthesized $\mathrm{Cd}$ particles and a $50 \%$ of fully reacted $\mathrm{CdS}$ hollow particles was used as a reference to determine the ratio of the x-ray diffraction factors for the mentioned lattice interspacings of $\mathrm{Cd}$ and $\mathrm{CdS}$. This ratio (0.275) was used to quantitatively follow the $\mathrm{Cd}$ to $\mathrm{CdS}$ conversion from the integrated areas: $[\mathrm{CdS}] /([\mathrm{Cd}]+[\mathrm{CdS}])=\mathrm{A}_{\mathrm{CdS}} /\left(0.275 \cdot \mathrm{A}_{\mathrm{Cd}}+\mathrm{A}_{\mathrm{CdS}}\right)$

TEM micrographs were obtained using a Tecnai G2 S-Twin electron microscope (200 $\mathrm{kV})$. TEM samples were prepared by placing a drop of the colloidal solution containing the nanoparticles onto a carbon-coated copper grid at room temperature and ambient atmosphere. Only the grid containing the initial cadmium nanoparticles (Fig. 1A) was

prepared and mounted in the TEM holder under an argon atmosphere. This sample was quickly transferred into the TEM, reducing its air exposure to around $20 \mathrm{~s}$. The in situ TEM analysis was conducted using a JEOL 3010 equipped with a heating sample holder. Samples were deposited on silicon nitride membranes and heated in an ultra high vacuum inside the TEM.

\section{Acknowledgements}

This work was supported by the Director, Office of Science, Office of Basic Energy Sciences, of the U.S. Department of Energy under Contract No. DE-AC02-05CH11231. The authors acknowledge support of the National Center for Electron Microscopy at 
Lawrence Berkeley National Laboratory, which is also supported by the U.S. Department of Energy under Contract No. DE-AC02-05CH11231.

Supporting information available: Bright- and dark-field TEM micrographs with the corresponding electron diffraction patterns of the precursor $\mathrm{Cd}$ particles, TEM micrographs at different tilting angles of $\mathrm{Cd} / \mathrm{CdTe}$ heterostructures, Arrhenius plot of the diffusion coefficients for $\mathrm{Cd}, \mathrm{S}$, and other elements in CdS. This material is available free of charge via the Internet at http://pubs.acs.org. 


\section{References and Notes}

1. Yin, Y. D.; Rioux, R. M.; Erdonmez, C. K.; Hughes, S.; Somorjai, G. A.; Alivisatos, A. P. Formation of Hollow Nanocrystals Through the Nanoscale Kirkendall Effect. Science 2004, 304, 711-714.

2. Fan, H.-J.; Gösele, U.; Zacharias, M. Formation of Nanotubes and Hollow Nanoparticles Based on Kirkendall and Diffusion Processes: A Review. Small 2007, 3, 1660-1671.

3. Cabot, A.; Puntes, V. F.; Shevchenko, E.; Yin, Y.; Balcells, L.; Marcus, M. A.; Hughes, S. M.; Alivisatos, A. P. Vacancy Coalescence During Oxidation of Iron Nanoparticles. J. Am. Chem. Soc. 2007, 129, 10358-10359.

4. Liu, B.; Zeng, H. C. Fabrication of $\mathrm{ZnO}$ "Dandelions" via a Modified Kirkendall Process. J. Am. Chem. Soc. 2004, 126, 16744-16746.

5. Chiang, R.-K.; Chiang, R.-T. Formation of Hollow $\mathrm{Ni}_{2} \mathrm{P}$ Nanoparticles Based on the Nanoscale Kirkendall Effect. Inorg. Chem. 2007, 46, 369-371.

6. Peng, H. L.; Xie, C.; Schoen, D. T.; McIlwrath, K.; Zhang, X. F.; Cui, Y. Ordered Vacancy Compounds and Nanotube Formation in CuInSe ${ }_{2}-\mathrm{CdS}$ Core-Shell Nanowires. Nano Lett. 2007, 7, 3734-3738.

7. Fan, H.-J.; Knez, M.; Scholz, R.; Hesse, D.; Nielsch, K.; Zacharias, M.; Gösele, U. Influence of Surface Diffusion on the Formation of Hollow Nanostructures Induced by the Kirkendall Effect: The Basic Concept. Nano Lett. 2007, 7, 993-997.

8. Yin, Y. D.; Erdonmez, C. K.; Cabot, A.; Hughes, S.; Alivisatos, A. P. Colloidal Synthesis of Hollow Cobalt Sulfide Nanocrystals. Adv. Funct. Mater. 2006, 16, 13891399.

9. Wang, C. M.; Baer, D. R.; Thomas, L. E.; Amonette, J. E.; Antony, J.; Qiang, Y.; Duscher, G. Void Formation During Early Stages of Passivation: Initial Oxidation of Iron Nanoparticles at Room Temperature. J. Appl. Phys. 2005, 98, 094308.

10. Nakamura, R.; Lee, J. G.; Mori, H.; Nakajima, H. Oxidation Behaviour of Ni Nanoparticles and Formation Process of Hollow NiO. Philos. Mag. 2008, 88, 257-264.

11. Wang, Y. L.; Xia, Y. N. Bottom-Up and Top-Down Approaches to the Synthesis of Monodispersed Spherical Colloids of Low Melting-Point Metals. Nano Lett. 2004, 4, 2047-2050.

12. We acknowledge that as these structures are prepared in the solution phase at moderate temperatures and with evidence that these shells are not monocrystalline, there may not be a true void inside of these structures - surfactant and solvent may be present within.

13. Mehrer, H.; Stolica, N.; Stolwijk, N. A., Diffusion in Solid Metals and Alloys. In Landolt-Börnstein, Group III, Springer-Verlag: Berlin, 1990; Vol. 26, Chapter 2.

14. Meyer, B. Elemental Sulfur. Chem. Rev. 1976, 76, 367-388.

15. Cabrera, N.; Mott, N. F. Theory of the Oxidation of Metals. Rep. Prog. Phys. 1948, $12,163-184$.

16. Hermans, J. J. Diffusion with Discontinuous Boundary. J. Coll. Sci. 1947, 2, 387398.

17. Horváth, J.; Birringer, R.; Gleiter, H. Diffusion in Nanocrystalline Material. Solid State Commun. 1987, 62, 319-322. 
18. Fromhold, J., A. T. Theory of Metal Oxidation. North-Holland: Amsterdam, 1976; Vol. 1, p 275.

19. Fehlner, F. P. Low-Temperature Oxidation. Wiley-Interscience: 1986; p 71.

20. Dutt, M. B.; Sharma, B. L., Diffusion in Compound Semiconductors. In LandoltBörnstein, Group III, Springer-Verlag: Berlin, 1998; Vol. 33A, Chapter 3.

21. Woodbury, H. H. Diffusion of Cd in CdS. Phys. Rev. A 1964, 134, A492-A498.

22. Shaw, D. The Self-Diffusion of Cd in CdS. J. Phys. C. 1984, 17, 4759-4782.

23. Jones, E. D. Measurement of Self-Diffusion of Cadmium into Cadmium Sulfide Using Radiotracer Techniques. J. Phys. Chem. Solids 1972, 33, 2063-2069.

24. Kumar, V.; Kröger, F. A. Self-Diffusion and Defect Structure of Cadmium Sulfide. J. Solid State Chem. 1971, 3, 387-400.

25. Jones, E. D.; Stewart, N. M.; Thambipillai, V. Self-Diffusion of Cadmium into Cadmium Sulfide. J. Cryst. Growth 1989, 96, 453-455.

26. Sysoev, L. A.; Gelfman, A. J.; Kovaleva, A. D.; Kravchenko, N. G. Izv. Akad. Nauk SSSR Neorg. Mater. 1969, 5, 2208. 


\title{
SUPPORTING INFORMATION
}

\section{Sulfidation of Cadmium at the Nanoscale}

\author{
Andreu Cabot, ${ }^{1}$ Rachel K. Smith, ${ }^{1}$ Yadong Yin, ${ }^{2}$ Haimei Zheng, ${ }^{1}$ Björn M. \\ Reinhard, ${ }^{3}$ Haitao Liu, ${ }^{4}$ and A. Paul Alivisatos ${ }^{1,4} *$ \\ ${ }^{1}$ Materials Sciences Division, Lawrence Berkeley National Laboratory, Berkeley, CA 94720. \\ ${ }^{2}$ Department of Chemistry, University of California at Riverside, Riverside, CA 92521. \\ ${ }^{3}$ Department of Chemistry, Boston University, Boston, MA 02215. \\ ${ }^{4}$ Department of Chemistry, University of California at Berkeley, Berkeley, CA 94720.
}

Solid cadmium particles were synthesized by the decomposition of dimethylcadmium in trioctylphosphine oxide (TOPO) at $330^{\circ} \mathrm{C}$ under air-free conditions. The resulting $\mathrm{Cd}$ nanoparticles are monocrystalline, as deduced from the electron diffraction and dark field TEM micrographs shown in Fig. S.I. 1.
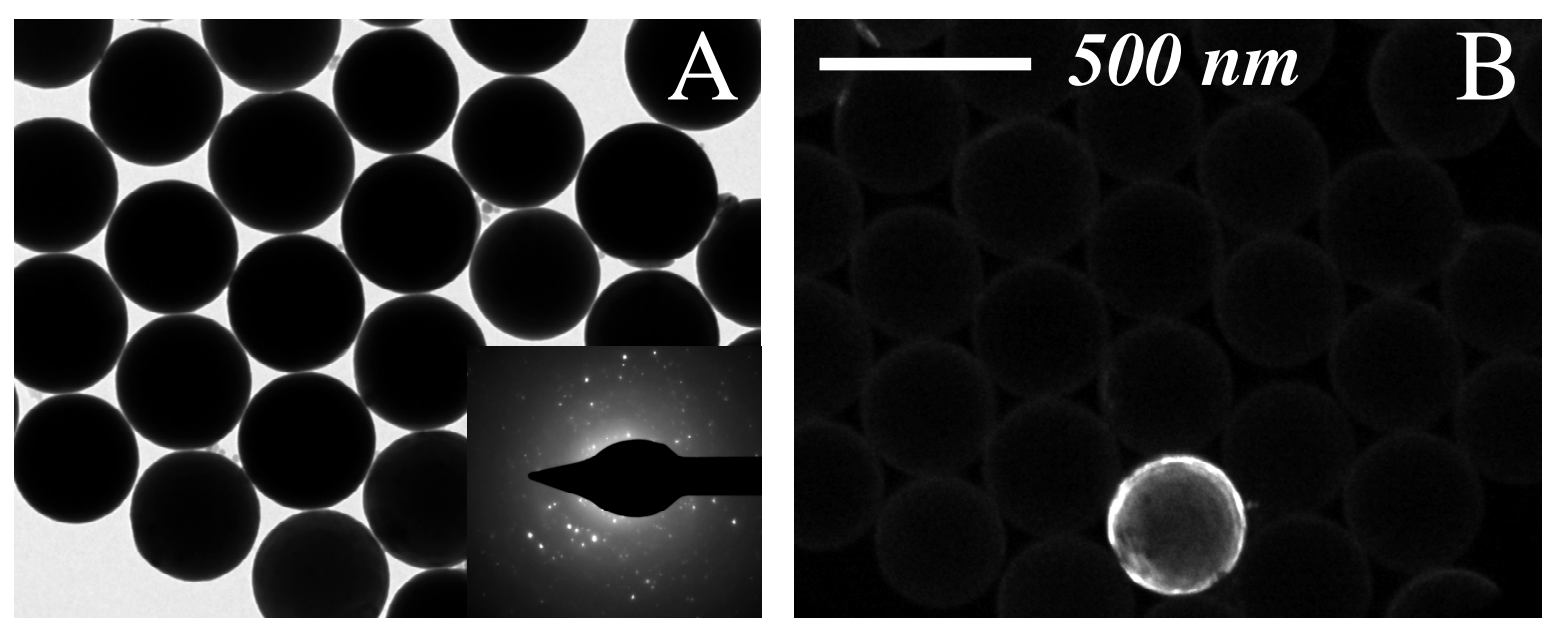

Fig. S.I. 1 Bright (A) and dark (B) field TEM micrograph of the precursor cadmium particles. The electron diffraction pattern of the group of particles shown on A is shown as an inset on the bottom-right corner of the micrograph. The dark field TEM micrographs shown in B is obtained by selecting just one of the diffraction spots on the electron diffraction pattern. 
Our studies reveal important differences in the morphologies of surface supported and liquid phase metal-void structures and underline the need to consider the effect of the support in comparisons between colloidal and supported particles. When the Cd particles are supported on $\mathrm{SiN}$ membranes and are submerged into a solution containing sulfur, the intermediate $\mathrm{Cd}$ cores stay close to the middle of the particle, appearing highly faceted with mostly hexagonal geometries (Fig. S.I. 2). Under these conditions, the observed kinetics are very similar to the reaction of free particles in solution. However, the substrate plays an important role in determining the most thermodynamically stable structure.

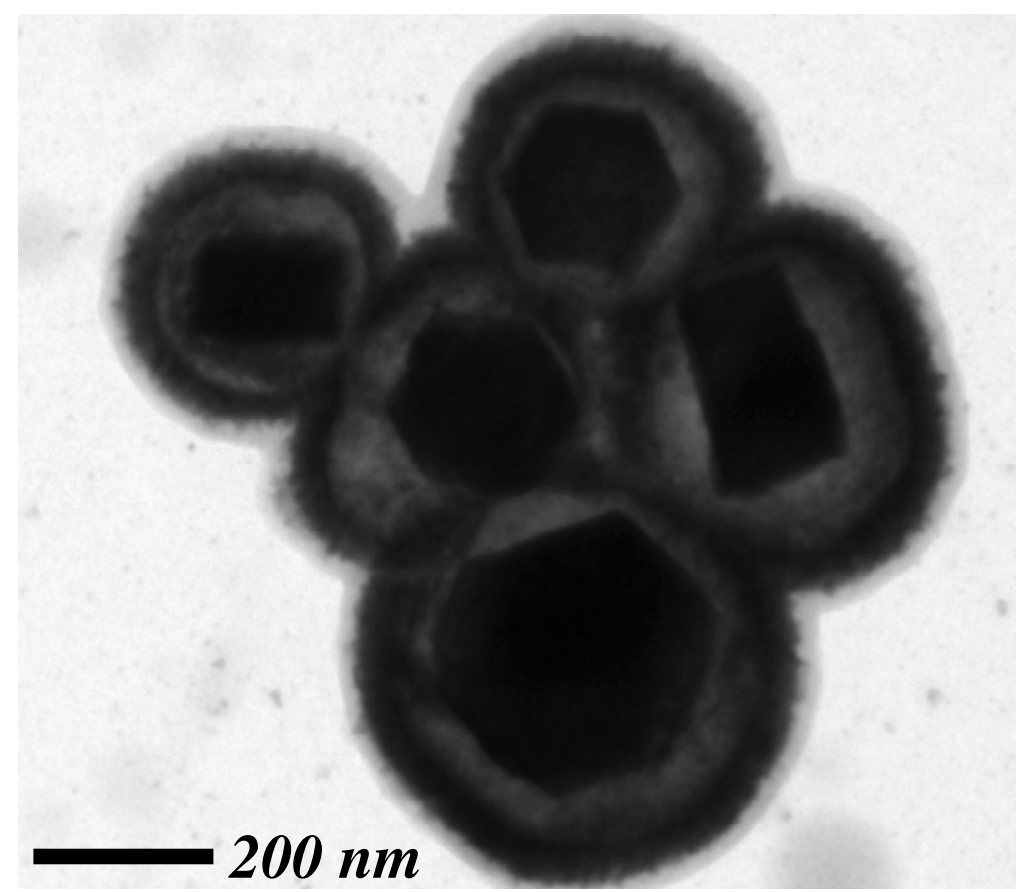

Fig. S.I. 2 TEM micrographs of partially reacted $\mathrm{Cd} / \mathrm{CdS}$ particles while supported on a silicon nitride grid. 
When reacting Cd particles with trioctylphosphine telluride (TOPTe), the resulting CdTe has a higher porosity than the CdS. At the same time the crystal grain domains partially arrange in a columnar structure, offering fast paths for ion diffusion. In such a shell, the $\mathrm{Cd}$ diffusion is very fast. This high diffusivity, in combination with the high Cd-TOPTe reactivity, results in a very large shell growth rate. Similar to the sulfidation of $\mathrm{Cd}$, the CdTe reaction also involves a net outward flow of material leading to the formation of core-void-shell structures. However, in contrast to the $\mathrm{Cd} / \mathrm{CdS}$ case, some $\mathrm{Cd} / \mathrm{CdTe}$ heterostructures contain approximately spherical $\mathrm{Cd}$ cores after reaction at high temperatures $\left(\sim 300^{\circ} \mathrm{C}\right.$, Fig. S.I. 3). This observation points towards a similar time scale for $\mathrm{Cd}$ /vacancy self-diffusion inside the particle and across the CdTe shell. The very fast growth of the CdTe shell pulls out $\mathrm{Cd}^{2+}$ and injects vacancies at a very fast rate, resulting in a kinetic control of the core shape.

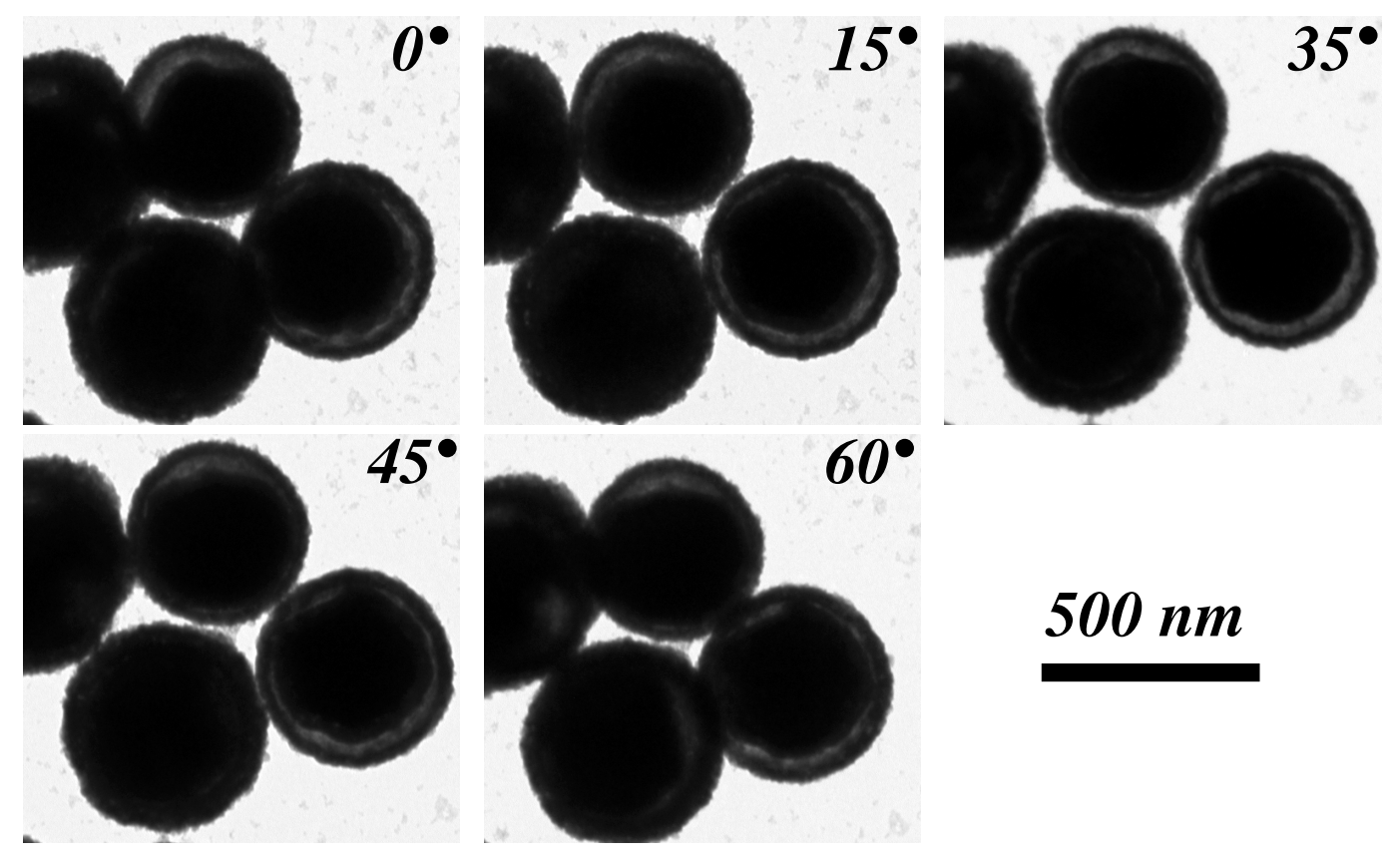

Fig. S.I. 3 TEM micrographs at different tilting angles of $\mathrm{Cd} / \mathrm{CdTe}$ heterostructures. 


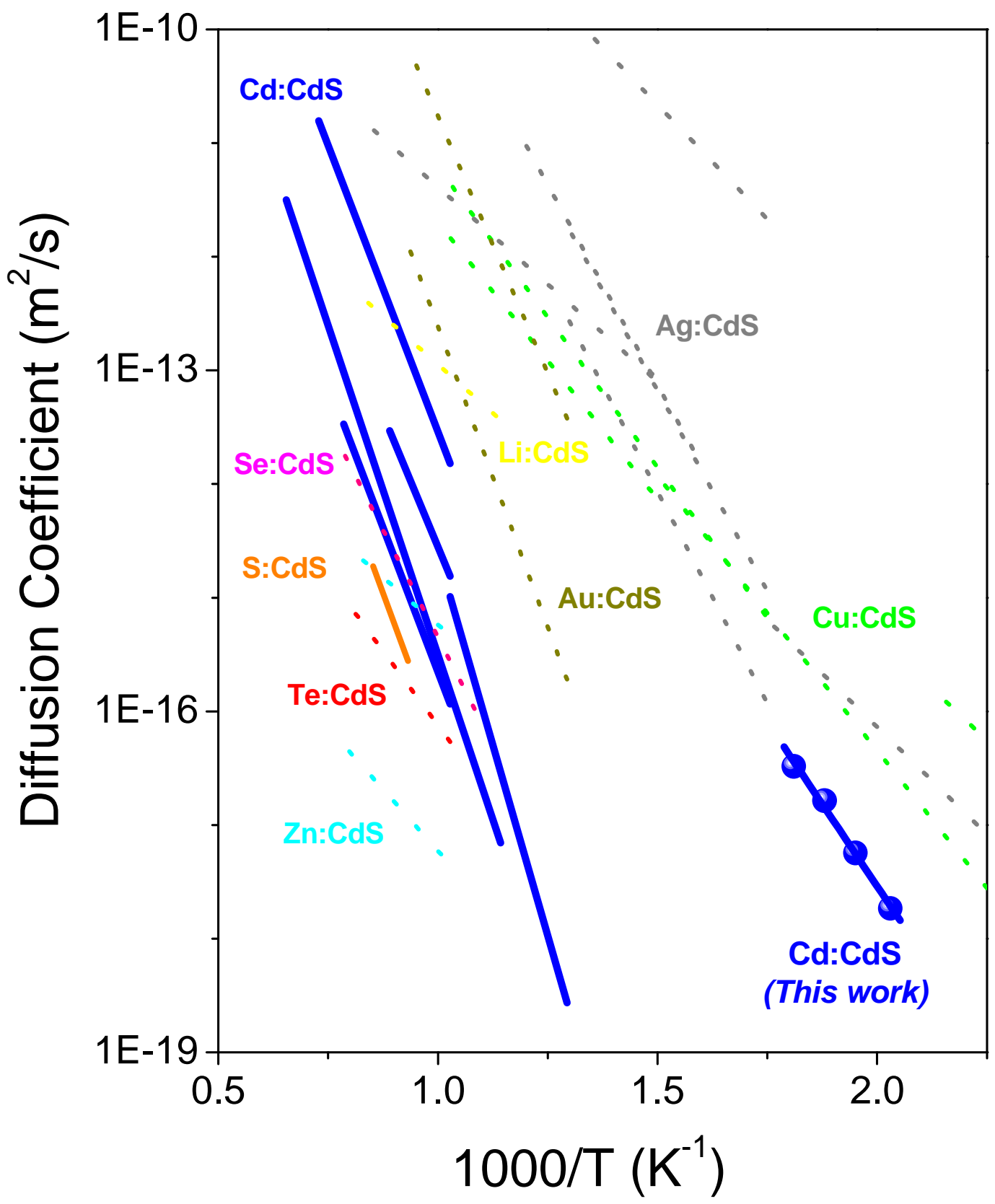

Fig. S.I. 4 Arrhenius plot of the literature diffusion coefficients for $\mathrm{Cd}, \mathrm{S}$ and other elements in CdS. 


\section{DISCLAIMER}

This document was prepared as an account of work sponsored by the United States Government. While this document is believed to contain correct information, neither the United States Government nor any agency thereof, nor The Regents of the University of California, nor any of their employees, makes any warranty, express or implied, or assumes any legal responsibility for the accuracy, completeness, or usefulness of any information, apparatus, product, or process disclosed, or represents that its use would not infringe privately owned rights. Reference herein to any specific commercial product, process, or service by its trade name, trademark, manufacturer, or otherwise, does not necessarily constitute or imply its endorsement, recommendation, or favoring by the United States Government or any agency thereof, or The Regents of the University of California. The views and opinions of authors expressed herein do not necessarily state or reflect those of the United States Government or any agency thereof or The Regents of the University of California. 